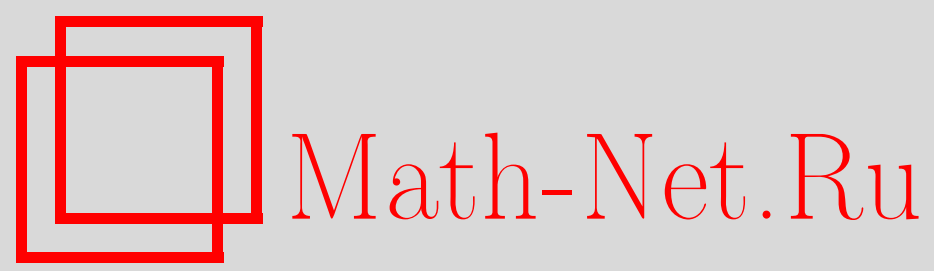

В. С. Лубенцова, А. В. Ефремов, Моделирование производственной программы автотранспортного предприятия с использованием марковских случайных процессов, Вестн. Сам. гос. техн. ун-та. Сер. Физ.-мат. науки, 2002, выпуск 16, 155-160

DOI: https://doi.org/10.14498/vsgtu114

Использование Общероссийского математического портала Math-Net.Ru подразумевает, что вы прочитали и согласны с пользовательским соглашением

http://www.mathnet.ru/rus/agreement

Параметры загрузки:

IP : 3.85 .7 .115

26 апреля 2023 г., 10:29:15 


\title{
МОДЕЛИРОВАНИЕ ПРОИЗВОДСТВЕННОЙ ПРОГРАММЫ АВТОТРАНСПОРТНОГО ПРЕДПРИЯТИЯ С ИСПОЛЬЗОВАНИЕМ МАРКОВСКИХ СЛУЧАЙНЫХ ПРОЦЕССОВ
}

\begin{abstract}
Проведён анализ прочесса эксплуатации группь автомобилей как случайного процесса с дискретными состояниями и непрерывным временем (пробегом). Приведены результаты расчётов основных показателей производственной программы автотранспортного предприятия (АТП): коэффициента выпуска автомобиля, коэффиџиента технической готовности, годового пробега автомобиля и провозных возможностей АТП.
\end{abstract}

Развитие рыночных отношений в экономике обусловливает необходимость перехода к принципам управления, наиболее соответствующим современным условиям, принципам логистики, дающим возможность гибкого реагирования производственных и торговых систем на быстро изменяющиеся приоритеты потребителей.

Принципиальная новизна логистического подхода к управлению АТП состоит в том, что оно рассматривается как внутрипроизводственная логистическая система на макро- и микроуровне. На макроуровне АТП выступает в качестве элемента макрологистических систем. На этом уровне АТП обеспечивают ритм работы этих систем, являясь источниками транспортных услуг. На микроуровне АТП представляет собой совокупность подсистем, находящихся в отношениях и связях друг с другом и образующих целостность. Эти подсистемы обеспечивают вхождение материального потока в микрологистическую систему, прохождение внутри неё и выход из системы в виде транспортных услуг.

Моделирование производства транспортных услуг, его материального обеспечения и надежности работы подвижного состава, участвующего в данном производстве, необходимо рассматривать как единый процесс, обеспечивающий получение достоверной оценки возможностей АТП по созданию конкурентоспособной продукции (транспортных услуг).

Основными итоговыми показателями расчёта производственной программы по эксплуатации подвижного состава являются коэффициент технической готовности, коэффициент выпуска, годовые пробеги автомобилей и провозные возможности АТП. Величина провозных возможностей АТП в значительной степени зависит от коэффициента выпуска автомобилей.

Коэффициент выпуска автомобилей, по существу, является вероятностью безотказной работы автомобилей. Действительно, вероятность безотказной работы - вероятность того, что в пределах заданной наработки отказ объекта не возникнет. Статистически вероятность безотказной работы $P(t)$ определяется отношением количества оставшихся к моменту наработки $\mathrm{t}$ работоспособных объектов $N(t)$ к общему числу объектов $N(O)$ :

$$
P(t)=\frac{N(t)}{N(0)}=1-\frac{N(0)-N(t)}{N(0)}=1-\frac{r(t)}{N(0)},
$$

где $r(t)$ - количество отказавших объектов к моменту $t$.

Коэффициент выпуска автомобилей представляет собой отношение количества эксплуатирующихся к моменту $t$ автомобилей $A_{\ni}$ к общему количеству автомобилей в парке $A_{\text {сп }}$ :

$$
\alpha_{\mathrm{B}}=\frac{A_{\ni}}{A_{\text {сп }}}=1-\frac{A_{\text {сп }}-A_{\ni}}{A_{\text {сп }}}=1-\frac{A_{\text {пр }}}{A_{\text {сп }}},
$$

где $A_{\text {пр }}$ - количество автомобилей, которые к моменту $t$ простаивают по различным причинам (находятся в ремонте, техническом обслуживании, без водителя, без шин; бездорожье и др.).

Простое сравнение формул (1) и (2) позволяет говорить о том, что коэффициент выпуска автомобилей и вероятность безотказной работы автомобилей - понятия адекватные.

Для моделирования коэффициента выпуска автомобилей воспользуемся аппаратом марковских дискретных случайных процессов с непрерывным временем. Представим автомобиль как некоторую систему $S$ с дискретными состояниями $S_{0}, S_{1}, \ldots, S_{n}$, которая переходит из состояния в состояние под влиянием случайных событий (отказов). На стадии планирования работы автомобиля целесообразно рассматривать следующие состояния, в которых он может находиться в процессе эксплуатации, и которые характеризуются целодневными простоями: $S_{0}$ - исправен, работает; $S_{1}$ - находится в капитальном ремонте (КР); $S_{2}$ - проходит ТО-2; $S_{3}-$ находится в текущем ремонте (ТР); $S_{4}$ - исправен, не работает по организационным причинам 
(без водителя, без шин, без запасных частей); $S_{5}-$ не работает, снятие агрегата для отправки в капитальный ремонт; $S_{6}-$ не работает, списание агрегата, замена на новый; $S_{7}-$ исправен, не работает (выходные и праздничные дни); $S_{8}$ - списывается.

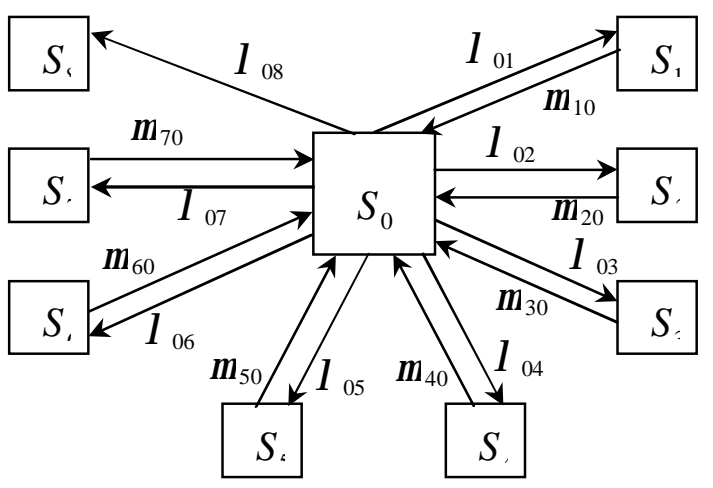

Р и с . 1. Граф состояний автомобиля

Для анализа процесса эксплуатации автомобиля как случайного процесса построим граф состояний автомобиля (рис. 1).

Рассматриваемые состояния автомобиля $S_{j}$ характеризуются средним числом дней пребывания автомобиля в каждом состоянии $Д_{j}$. Показатели $Д_{j}$ находят отражение в статистической отчётности АТП. Отношение

$$
P_{j}=\frac{Д_{j}}{Д_{k}},
$$

где $Д_{k}-$ число календарных дней в году, можно трактовать как вероятность нахождения автомобиля в $j$-том состоянии.

Вероятности состояний автомобиля $P_{1}, \ldots, P_{j}, \ldots, P_{n}$ как функции пробега в случае непрерывных марковских цепей удовлетворяют уравнениям Колмогорова, записываемым в виде

$$
\left\{\begin{array}{l}
\frac{d P_{0}(L)}{d L}=-l_{c} \sum_{i=1}^{n} \lambda_{0 i}(L) P_{0}(L)+\sum_{i=1}^{n-1} \mu_{i 0}(L) P_{i}(L) ; \\
\frac{d P_{i}(L)}{d L}=l_{c} \lambda_{0 i}(L) P_{0}(L)-\mu_{i 0}(L) P_{i}(L), \quad i=\overline{1, n-1} ; \\
\frac{d P_{n}(L)}{d L}=l_{c} \lambda_{0 n}(L) P_{0}(L),
\end{array}\right.
$$

где $P_{i}(L)$ - вероятность нахождения автомобиля в $i$-том состоянии $(i=\overline{0, n}) ; \lambda_{0 i}(L)-$ интенсивность перехода автомобиля из нулевого в $i$-тое состояние; $\mu_{i 0}(L)$ - интенсивность перехода автомобиля из $i$-того в нулевое состояние; $l_{c}-$ коэффициент, отражающий связь между наработками в днях и километрах пробега (среднесуточный пробег). Вероятность нахождения автомобиля в состоянии “исправен, работает" $P_{0}(L)$ представляет собой коэффициент выпуска $\alpha_{\mathrm{B}}(L)$, а сумма вероятностей $P_{0}(L)+P_{4}(L)+P_{7}(L)$ - коэффициент технической готовности автомобиля $\kappa_{\text {тг }}$.

Согласно расчётам, проведённым на основе статистических данных эксплуатации автомобиля, все потоки, переводящие автомобиль из состояния в состояние, являются пуассоновскими или сводятся к ним путём рассмотрения процесса эксплуатации на малых интервалах пробега (1 - 2 тыс. км) и корректировки исходного потока отказов деталей для исключения последействия. Таким образом, процесс, протекающий в системе, является марковским.

В таблице приведены формулы интенсивностей перехода для расчёта $\alpha_{в}(L)$ и $\kappa_{\text {тг }}$

Нужно учесть, что для расчёта производственной программы АТП необходимо зачастую определять показатели работы группы автомобилей определённой модели $j$ - возраста (коэффициент выпуска и годовой пробег автомобиля $j$ - возрастной группы). Для описания процесса функционирования группы автомобилей может быть использован метод динамики средних. Удобство его заключается в том, что, зная возможные состояния одного (условного) автомобиля, можно моделировать процесс функционирования группы из любого числа автомобилей. Схема, изображающая процесс работы условного автомобиля, аналогична схеме на рис. 1 лишь с той разницей, что через $\lambda_{i j}$ и $\mu_{i j}$ обозначены средние интенсивности потоков событий, переводящих автомобиль из состояния $S_{i}$ в состояние $S_{j}$ и наоборот. При этом каждое состояние характеризуется средней численностью $N_{j}(t)$, находящихся в нём в момент времени $t$. Величина $N_{j}(t)$ для любого $t$ представляет собой случайную величину, а вообще, при меняющемся $t$ - случайную функцию времени. 
Интенсивности перехода $\lambda_{i j}$ и $\mu_{j i}$

\begin{tabular}{|c|c|c|}
\hline Интенсивность & Формула, принятая в расчёте & Примечание \\
\hline Исправен - капитальный ремонт & $\lambda_{01}(L)=\frac{1}{\sqrt{2 \pi} \sigma} e^{-\left(\frac{L-m}{\sigma}\right)^{2}}$ & $m=50, \sigma=5$ \\
\hline Исправен - проходит ТО-2 & $\lambda_{02}(L)=\left(\bar{L}_{\text {то }}\right)^{-1}$ & $\begin{array}{l}\bar{L}_{\text {то }}-\text { средняя периодич- } \\
\text { ность ТО- } 2 \text {, } \\
\bar{L}_{m o}=10 \text { mысс.км }\end{array}$ \\
\hline Исправен - находится в ТР & $\lambda_{03}(L)=\frac{F}{L_{\text {инт }}}$ & $\begin{array}{c}F-\text { число ДЛН автомобиля, } \\
F=35 \text { шт. }, \quad L_{\text {инт }}-\text { рас- } \\
\text { сматриваемый } \quad \text { интервал } \\
\text { пробега, } L_{\text {инт }}=100 \text { тыс. км }\end{array}$ \\
\hline $\begin{array}{l}\text { Исправен - простаивает по орга- } \\
\text { низационным причинам }\end{array}$ & $\lambda_{04}(L)=\left(l_{c} \bar{T}_{\text {пр }}\right)^{-1}$ & $\begin{array}{l}\bar{T}_{\text {пр }}-\text { среднее время между } \\
\text { простоями, } \bar{T}_{\text {пр }}=7 \text { дн.; } l_{c}- \\
\text { среднесуточный } \\
l_{c}=0,25 \text { пыс. км }\end{array}$ \\
\hline $\begin{array}{l}\text { Исправен - капитальный ремонт } \\
\text { агрегата }\end{array}$ & $\lambda_{05}(L)=\frac{A}{L_{\text {инт }}}$ & $\begin{array}{l}A-\text { число агрегатов автомо- } \\
\text { биля, } A=5 \text { шт. }\end{array}$ \\
\hline Исправен - списание агрегата & $\lambda_{06}(L)=\frac{A}{L_{\text {инт }}}$ & \\
\hline $\begin{array}{l}\text { Исправен - не работает (празд- } \\
\text { ничные и выходные дни) }\end{array}$ & $\lambda_{07}(L)=\left(l_{c} \bar{T}_{\text {вых }}\right)^{-1}$ & $\begin{array}{l}\bar{T}_{\text {вых }}-\text { среднее время между } \\
\text { простоями, } \bar{T}_{\text {вых }}=7 \text { дн. }\end{array}$ \\
\hline Исправен - списание автомобиля & $\lambda_{08}(L)=\left(L-L_{0}\right) / \delta^{2}$ & $\begin{array}{l}\delta^{2}=1600 \mathrm{mblc} . \kappa M \\
L_{0}=270 \mathrm{mblc.} . \kappa M, L>L_{0}\end{array}$ \\
\hline Капитальный ремонт - исправен & $\mu_{10}(L)=\left(\bar{T}_{\text {кр }}\right)^{-1}$ & $\begin{array}{l}\bar{T}_{\text {кр }}-\text { средняя продолжи- } \\
\text { тельность капитального ре- } \\
\text { монта, } \bar{T}_{\text {кр }}=20 \text { дн. }\end{array}$ \\
\hline ТО-2 - исправен & $\mu_{20}(L)=\left(\bar{T}_{\text {то }}\right)^{-1}$ & $\begin{array}{c}\bar{T}_{\text {то }}-\text { средняя } \text { продолжи- } \\
\text { тельность ТО- } 2, \bar{T}_{\text {то }}=1 \text { дн. }\end{array}$ \\
\hline Находится в ТР - исправен & $\mu_{30}(L)=\left(\bar{T}_{\mathrm{r}}\right)^{-1}$ & $\begin{array}{l}\bar{T}_{\text {т }}-\text { средняя } \quad \text { продолжи- } \\
\text { тельность ТР, } \bar{T}_{\text {т }}=1 \text { дн. }\end{array}$ \\
\hline $\begin{array}{l}\text { Простаивает по организационным } \\
\text { причинам - исправен }\end{array}$ & $\mu_{40}(L)=\left(\bar{T}_{\text {п }}\right)^{-1}$ & $\begin{array}{lr}\bar{T}_{\text {п }} \text { средняя } & \text { продолжи- } \\
\text { тельность } & \text { простоя, } \\
\bar{T}_{\text {п }}=1 \text { дн. } & \\
\end{array}$ \\
\hline $\begin{array}{l}\text { Капитальный ремонт агрегата - } \\
\text { исправен }\end{array}$ & $\mu_{50}(L)=\left(\bar{T}_{\text {кр }}^{\mathrm{a}}\right)^{-1}$ & $\begin{array}{l}\bar{T}_{\kappa p}^{a}-\text { средняя продолжи- } \\
\text { тельность простоя при сня- } \\
\text { тии агрегата, } \bar{T}_{\text {кр }}^{\mathrm{a}}=1 \text { дн. }\end{array}$ \\
\hline Списание агрегата - исправен & $\mu_{60}(L)=\left(\bar{T}_{\mathrm{p}}^{\mathrm{c}}\right)^{-1}$ & $\begin{array}{l}\bar{T}_{\mathrm{p}}^{\mathrm{c}}-\text { среднее время замены } \\
\text { агрегата, } \bar{T}_{\mathrm{p}}^{\mathrm{c}}=1 \text { дн. }\end{array}$ \\
\hline $\begin{array}{l}\text { Исправен, не работает (празд- } \\
\text { ничные и выходные дни) - испра- } \\
\text { вен, работает }\end{array}$ & $\mu_{70}(L)=\left(\bar{T}_{\text {пр }}\right)^{-1}$ & $\begin{array}{lr}\bar{T}_{\text {пр }}-\text { средняя } & \text { продолжи- } \\
\text { тельность } & \text { простоя, } \\
\bar{T}_{n p}=1 \text { дн. } & \end{array}$ \\
\hline
\end{tabular}


Аналогично случаю с одним автомобилем, для группы автомобилей все потоки, переводящие условный автомобиль из состояния в состояние, с достаточной степенью точности согласуются с пуассоновскими, и система дифференциальных уравнений для средних численностей состояний принимает следующий вид:

$$
\left\{\begin{array}{l}
\frac{d N_{0}(L)}{d L}=-l_{c} \sum_{i=1}^{n} \lambda_{0 i}(L) N_{0}(L)+\sum_{i=1}^{n-1} \mu_{i 0}(L) N_{i}(L) ; \\
\frac{d N_{i}(L)}{d L}=l_{c} \lambda_{0 i}(L) N_{0}(L)-\mu_{i 0}(L) N_{i}(L), \quad i=\overline{1, n-1} ; \\
\frac{d N_{n}(L)}{d L}=l_{c} \lambda_{0 n}(L) N_{0}(L) .
\end{array}\right.
$$

Отношение $N_{0}(L) / N$ равно коэффициенту выпуска автомобилей определённой модели на пробеге $L$ с начала их эксплуатации, а отношение $\left(N_{0}(L)+N_{4}(L)+N_{7}(L)\right) / N$ - коэффициенту технической готовности автомобилей.

В результате исследования группы из 16 автомобилей одной модели и возраста были получены следующие результаты (на конец исследуемого интервала пробега): $\alpha_{\text {в }}=0,653$; $\kappa_{\mathrm{тг}}=P_{0}+P_{4}+P_{7}=0,839$. Графики их поведения приведены на рис. $2,3$.

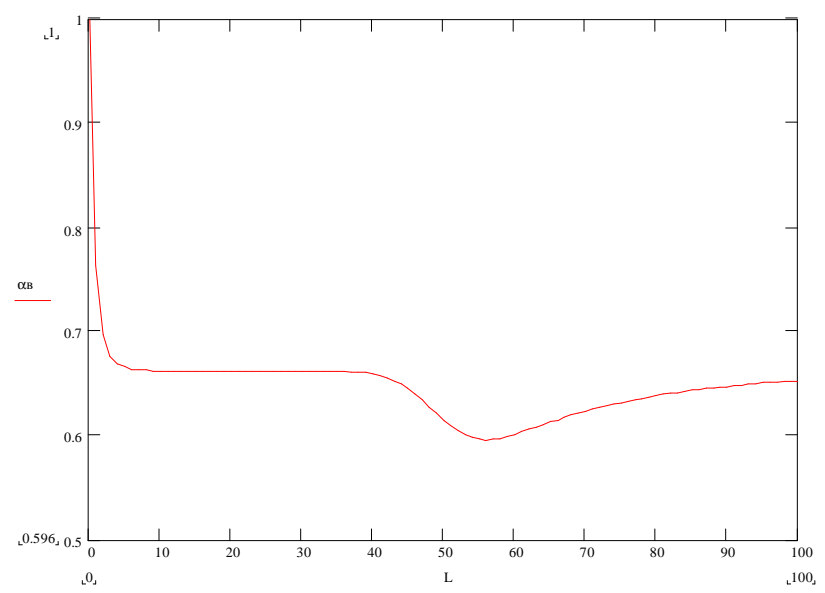

Р и с . 2. График зависимости коэффициента выпуска от величины пробега

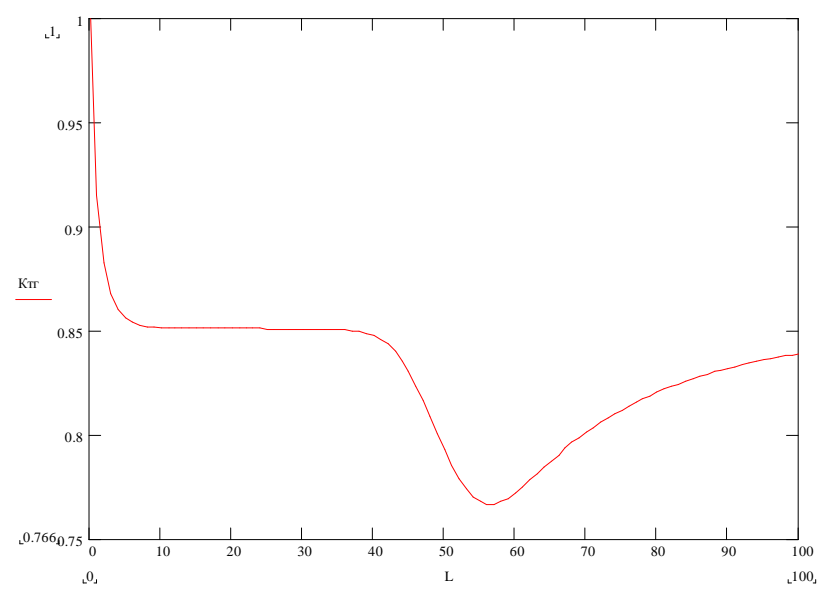

Р и с . 3. График зависимости коэффициента технической готовности от величины пробега

В ходе решения системы дифференциальных уравнений определяется коэффициент выпус-

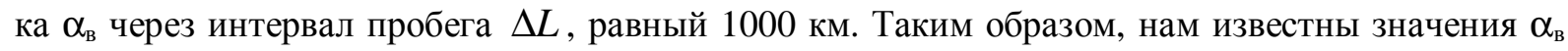
на начало года и через каждую тысячу километров пробега в течение года для автомобилей каждой $j$-той возрастной группы:

$$
\alpha_{\mathrm{B}}\left(L_{0 j}\right), \alpha_{\mathrm{B}}\left(L_{0 j}+\Delta L\right), \alpha_{\mathrm{B}}\left(L_{0 j}+2 \Delta L\right), \ldots, \alpha_{\mathrm{B}}\left(L_{0 j}+k \Delta L\right), \ldots,
$$

где $j=1, \ldots, h, k=1,2, \ldots$.

Период рабочего времени ДР, за который автомобиль выполнит пробег, равный 1000 км, равен

$$
Д P=\Delta L / l_{c} .
$$

К интервалов пробега, равных $\Delta L$, автомобиль выполнит за $Д K_{k j}$ календарных дней, определяемых формулой

$$
Д K_{k j}=\sum_{k=1}^{K} \Delta Д K_{k j},
$$

где $\Delta Д K_{k j}$ - период календарного времени, в течение которого автомобиль $j$-той возрастной группы реализует $k$-тый по счету интервал пробега, равный $\Delta L$. 
Годовой пробег автомобиль выполняет ДК календарных дней, ДК = 365 дн. Допустим, что на 365-й день величина годового пробега автомобиля попадает в $K$ - тый по счету интервал $\Delta L$ (рис. 4).

Годовой пробег автомобиля $L_{\text {гј }}$ (см. рис. 4$)$ складывается из $(K-1)$ интервалов $\Delta L$ и величины $\Delta l_{j}$, соответствующей какой-то части $K$ того по счету интервала $\Delta L$ :

$$
L_{\mathrm{r} j}=(K-1) \Delta L+\Delta l_{j},(9)
$$

Определим величину $\Delta l_{j}$. Для этого рассчитаем число

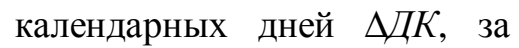
которые автомобиль выполнит пробег $\Delta l_{j}$ :

$$
\begin{gathered}
\Delta Д K_{j}=Д К-Д K_{(K-1) j}, \\
\text { где } Д K_{(K-1) j}=\sum_{k=1}^{K-1} \Delta Д K_{k j} .
\end{gathered}
$$

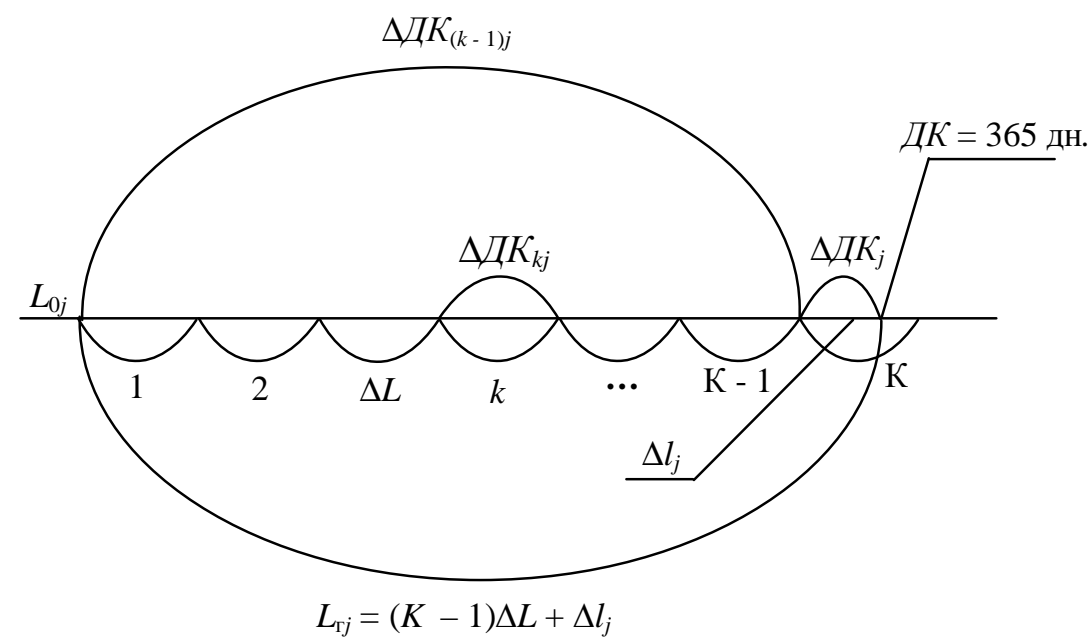

Р и с . 4. Временная эпюра случайной ситуации, сложившейся при определении годового пробега $L_{\text {гj }}$ автомобиля $j$-той возрастной группы.

Пробег автомобиля $\Delta l_{j} j$-того возраста за период $\Delta Д K_{j}$ определяется по формуле

$$
\Delta l_{j}=\Delta Д K_{j} l_{c}\left(\alpha_{\mathrm{B}}\left(L_{0 j}+(K-1) \Delta L\right)+\alpha_{\mathrm{в}}\left(L_{0 j}+K \Delta L\right)\right) / 2 .
$$

Расчет производится для всех возрастных групп каждой модели подвижного состава. Этот способ определения годового пробега позволяет снизить ошибку, получаемую в ходе аппроксимации данных $\alpha_{\text {в }}$ на 10 - 16\%, и тем самым повысить достоверность прогнозов годового пробега.

Для условного автомобиля годовой пробег составил $L_{\mathrm{r}}=59,51$ тыс. км.

Провозные возможности автотранспортного предприятия определяются по формулам

$$
\begin{gathered}
P=\sum_{i=1}^{n} \sum_{j=1}^{m} q_{i} \gamma_{i} \beta_{i} L_{i j} \\
Q=\sum_{i=1}^{n} \frac{P_{i}}{l_{\mathrm{cp} i}},
\end{gathered}
$$

где $L_{i j}$ - годовой пробег автомобиля $j$-того возраста $i$-той модели, тыс. км; $q_{i}$ - грузоподъемность автомобиля $i$-той модели, т; $\gamma_{i}-$ коэффициент использования грузоподъемности автомобиля $i$-той модели; $\beta_{i}$ - коэффициент использования пробега автомобиля $i$-й модели; $l_{\text {срi }}-$ среднее расстояние перевозки тонны груза, тыс. км; $P_{i}$ - грузооборот, выполняемый автомобилями $i$-той модели, тыс. ткм; $Q$ - объем перевозок АТП, тыс. т; $P$ - грузооборот АТП, тыс. ткм.

Для исследуемой группы автомобилей эти показатели составили $P=685551$ ткм; $Q=22852$ т при следующих значениях коэффициентов: $q=2 \mathrm{~T} ; \quad \gamma=0,9 ; \quad \beta=0,4$; $l_{\text {срi }}=30$ тыс. км.

Результаты прогноза коэффициентов выпуска, годовых пробегов, провозных возможностей служат основанием для расчета остальных показателей производственной программы по эксплуатации подвижного состава. Данный подход к прогнозированию основных показателей производственной программы обеспечивает комплексный учет надежности и интенсивности эксплуатации подвижного состава, позволяет отказаться от циклового метода расчета показателей производственной программы. 


\section{БИБЛИОГРАФИЧЕСКИЙ СПИСОК}

1. Лукинский В.С., Бережной В.И., Бережная Е.В., Цвиринько И.А. Логистика автомобильного транспорта: концепция, методы, модели. М.: Финансы и статистика, 2000. 280 с.

2. Смехов А.А. Основы транспортной логистики. М.: Транспорт, 1995. 197 с.

3. Сергеев В.И. Менеджмент в бизнес-логистике. М.: Филинъ, 1997. 772 с.

4. Сергеев В.И. Логистика в бизнесе. М.: ИНФРА-М, 2001. 608 с. 